\title{
Self-management: keeping it simple with "Flo"
}

This article was published in the following Dove Press journal:

Nursing: Research and Reviews

2 March 2015

Number of times this article has been viewed

\section{Audrey Cund ${ }^{1-4}$ \\ Jayne L Birch-Jones $1,3,4$ \\ Martin Kay' \\ Patricia Connolly ${ }^{4}$}

'School of Health, Nursing and Midwifery, University of The West of Scotland, Paisley, Scotland, UK; ${ }^{2}$ Nottinghamshire Health and Social Care Assistive Technology Programme, Mansfield and Ashfield Clinical Commissioning Group, National Health Service, Rainworth, Mansfield, Nottinghamshire, UK; ${ }^{3}$ Kay Consultancy Ltd, Newark, UK; ${ }^{4}$ Department of Biomedical Engineering, University of Strathclyde, Glasgow, Scotland, UK
Correspondence: Audrey Cund University of West of Scotland, School of Health, Nursing and Midwifery, A Block room A616, High Street, Paisley PAI 2BE, Scotland, UK

Tel +44 I4I 849 42 I।

Email audrey.cund@uws.ac.uk
Background: Living with and managing a long-term condition are complex processes that are further compounded by a range of medical and psychosocial factors. The size and scale of long-term conditions in the UK is vast, with strategic drivers seeking to find innovative ways to managing this problem.

Methods: This project introduced Simple Telehealth "Florence," a software-based short message service texting system to monitor a variety of conditions, such as diabetes, hypertension, and wounds, initially in one region of the East Midlands, England, UK. This article discusses the findings from our patient and staff evaluations of using the system. In total, 37 patients across four conditions, diabetes, chronic obstructive pulmonary disease, hypertension, and Asperger's syndrome, evaluated the study and this exercise involved 33 nurses in teams across three locations over a 6-month period. Evaluation was made using a simple, self-completed questionnaire and analysis of qualitative comments.

Results: The majority of patients find the service easy to use, reassuring, and reducing the number of contacts they had with their doctor. The results also show that staff did notice a reduction in consultation time, highlighting the potential of the system to save time; the majority viewed it as a support to existing approaches aimed at the management of long-term conditions.

Discussion: This study adds to the growing body of evidence that supports community nurses through the use of innovative ways to assess, support, and monitor long-term conditions. It also offers insight into the experience of patients and staff working together in one region of England, UK.

Recommendations: Embedding Simple Telehealth "Florence" as an option within primary care services in the UK is hereby proposed. This would add to the range of options and therapeutic approaches available through modern Telehealth.

Keywords: self-management, simple telehealth, Flo, patient experience, community nurse

\section{Introduction}

A rise in the number of long-term conditions and an aging population have been cited over the past decade as the key drivers and imperatives for change in health and social care services in the UK. ${ }^{1}$ A number of strategies to reduce the burden of long-term illnesses have been driving forward innovations across the UK. Examples of these include the Expert Patients Programmes, ${ }^{2}$ the use of community matrons, ${ }^{3}$ and the use of Telehealth and Telecare to assess, diagnose, and monitor conditions at home or at a distance from a health and social care provider. ${ }^{4-6}$

\section{Self-management}

Living with and managing a long-term condition comprise a complex process and this is compounded by multiple medical and psychosocial variables that disrupt the 
ebb and flow of care for patients, carers, and clinicians. ${ }^{7}$ Self-management is cited as an approach to improve the health outcomes of patients with long-term conditions ${ }^{2}$ and to reduce costs to the National Health Service (NHS). ${ }^{8}$ Lorig and Holman ${ }^{9,10}$ refer to self-management as the way in which an individual manages his or her illness as directed by a health professional. The emphasis in this relationship is on the interaction or the patient's ability to comply rather than the impact or effect of self-management.

Contemporary nursing literature supports a move away from traditional clinician-centered approaches and promotes the ideology of self-management involving a shared responsibility between patient and provider. ${ }^{11-13}$ Achieving this has proved challenging for nurses, with a number of studies highlighting the barriers and facilitators to self-management.

For example, Wilson et al ${ }^{12}$ examined nurses' role in chronic disease management and highlighted a number of themes, including vulnerability to litigation arising from empowering their patients to self-manage. The study also showed that the nurses had feelings of discomfort when working with expert patients, and this related to their perceived knowledge and confidence to respond to the patient's needs. Interestingly, the nurses felt that the expert patient would take up more of their time because they would be asking more questions and entering into discussion about treatments. Shen et $\mathrm{al}^{14}$ also in their qualitative study cite the lack of time offered by a health professional as a barrier to self-management. Thompson and Clark ${ }^{7}$ add that more investment is needed in terms of self-management tools and resources, as well as training for nurses and doctors. They also highlight that the barriers to self-management are not clinician dominated and state that patients' social circumstances, support at home, and reluctance to take on responsibility are equal barriers to the success of self-management programs.

Facilitators for patients include family support and peer support, alongside the recognition of culture and diversity. ${ }^{14,15}$ Patients ask that health professionals keep their guidance simple and practical and that they take the time to ask for patients' opinion. McWilliam ${ }^{10}$ introduces the notion of the health professional "sharing the power" in the care relationship, thus allowing an interactive or co-constructed relationship to be developed.

The authors posit that the introduction of successful selfmanagement accepts the values and beliefs of the patient, and the nurses' role is to apply the principles of clinical safety, health promotion, and education, at the same time supporting the choices, risks, and decisions made by the individual in a properly assessed case-by-case basis. However, even in a modern community setting in which practitioners are seeking to implement best practice for their patients, it can be difficult to translate the findings of larger studies into local settings, and confidence needs to be gained in implementing new systems.

In this regard, the system studied and reported in this article, Simple Telehealth NHS Florence, or "Flo" as it is known within NHS groups, is a tool that can encourage effective and efficient nurse-patient interactions that support more self-management from patients in the longer term as the system is embedded in practice.

\section{Telehealth and self-management}

Telehealth is defined as "the remote exchange of data between a patient at home and their clinician(s) to assist in diagnosis and monitoring typically used to support patients with Long Term Conditions." ${ }^{16}$ A strong evidence base supports the use of Telehealth over the past 2 decades, ${ }^{17,18}$ and multiple authors concur that it improves access to services; it is easy to use, saves time, and increases patient satisfaction. ${ }^{19-21}$ Advances in science and technology have created flexible ways to support individuals at home and at a distance from their health care provider. ${ }^{22}$ Doughty ${ }^{23}$ outlines these advances and emphasizes the drive from consumers of health services for systems that are easy to use, readily accessible, and convenient, eg, smartphones and other small computerized devices. Young and Wilkins ${ }^{25}$ also add that health monitoring, wearable sensors, and wellness systems are currently promoted as mechanisms for individuals to check symptoms, self-manage, monitor lifestyles, and empower change in health behavior. Recent market trends indicate that since 2013, 8.6 trillion short message service (SMS) alerts were sent around the world, with 129 million of these sent and received from the UK. ${ }^{25}$ They also add that every month, 25 million visits are made to $>500$ UK health and well-being sites. ${ }^{25}$ This evidence is cited in a series of strategic documents as part of the government's diffusion strategy to acknowledge the value and importance of using a range of digital applications to improve the patient/ NHS interface. ${ }^{4,27}$

One of the simplest and most widely used communication systems is now SMS text. The Cochrane review by De Jongh et $\mathrm{al}^{28}$ assessed the evidence of using SMS text messages to support the monitoring of long-term illnesses, such as diabetes, asthma, and hypertension. The review concluded that there is low to moderate evidence to support the wider adoption of SMS against the outcomes of self-management and health outcomes and utilization. The analysis of each individual study is positive and benefits have been identified 
in relation to the primary outcome of an individual's ability to self-manage conditions such as diabetes.

This article adds to the growing body of knowledge in relation to strategies to support self-management and describes patient and nurse experience of using Florence Simple Telehealth (Flo), an approach that allows selfreporting of conditions and clinician interaction via SMS text to and from the patient's mobile telephone. In particular, we discuss here how a short evaluation can be used to check local conditions and responses to Flo and comment on how learning can be drawn from such local evaluations that support and add to the findings of larger studies.

\section{Introduction to "Flo" Simple Telehealth}

Simple Telehealth is an NHS-inspired and -owned Telehealth solution, developed within NHS Stoke. "Flo" is used by nurses and doctors in a variety of settings across England to enable patients to take up the responsibility for monitoring and managing their health condition, lifestyle, and/or treatment. ${ }^{29-31}$

Simple Telehealth Florence "Flo" is a software-based SMS texting system that presents an easy-to-use friendly interface for patients and clinicians. A Web-based platform for clinicians allows patients to be recruited by text using their mobile phone number. Protocols for monitoring of a variety of conditions, such as diabetes, chronic obstructive pulmonary disease (COPD), respiratory failure, and wound dressings, have been developed, and specialist protocols can be introduced in line with national guidance. ${ }^{29-31}$

The system operates by responding to health information sent and received by SMS from the patient (Table 1). For example, the dialogue for hypertension monitoring within Table 1 shows the short exchange of information between Flo and the patient on a daily basis by text. The boundary parameters set by the community nurse are supported and underpinned by national protocols. Each reading provided by the patient then triggers an appropriate message, warning, or clinician alert (Table 1). The local community nurse can then log in and check all blood pressure readings from patients she is monitoring. Those outside the preset limits are automatically highlighted in the patient list. More interventions can be made for these patients by Flo, eg, personal texts or telephone calls, according to local protocols. All of the information received by Flo can be graphically represented to show patterns and progress, thus allowing the community nurse to plan, prepare, and structure their time and consultation more effectively.
Table I Application of Flo in hypertension

I. Default parameters: systolic pressure $(80-134 \mathrm{mmHg})$; diastolic pressure $(60-84 \mathrm{mmHg})$

II. Daily reminder at 8 am (service message)

$8 \mathrm{am}$ : "Hi. Don't forget to take your blood pressure this morning and again this evening, and text it in. Text BP, then your reading, eg, BP I40 80. Thanks, Flo."

12 hours later, if the patient has not replied to the first message:

“Hi. I've noticed you haven't sent in your readings today. Could you please text in BP, then the readings. Thanks Flo."

III. Within normal range

"Your BP reading is normal. Flo."

IV. Systolic and diastolic pressures above desirable range

"Your blood pressure is high today. Follow the advice in your management plan, and take the readings again at your usual time. Thanks, Flo."

V. Systolic and diastolic pressures below desirable range "Your blood pressure is low today. Take it again, and follow the advice in your management plan. Take care, Flo."

VI. Breach message Triggered when the readings reach either $200 \mathrm{mmHg}$ (systolic) or $105 \mathrm{mmHg}$ (diastolic) or if the BP is below $70 \mathrm{mmHg}$ (systolic) or $50 \mathrm{mmHg}$ (diastolic): "Your BP is outside the safe range - so contact a doctor today, as agreed in your shared management plan. Take care, Flo."

Notes: Copyright (C) 2013. Reprinted with permission from University of The West of Scotland. Chambers R. Simple telehealth SMS texting service: Flo clinical protocols AIM for Health version 2. NHS Stoke-on-trent Clinical Commissioning Group. ${ }^{37}$

Abbreviations: BP, blood pressure; Flo, Simple Telehealth "Florence."

In the remainder of the article, we discuss some of the responses of the patients and community nurses to an evaluation of Flo by 37 patients and 37 staff in the East Midlands region of England, UK, and discuss implications for the wider use of Flo. The evaluation highlights that it is possible for new teams to roll out systems such as Simple Telehealth Florence under small-scale pilot control to gain experience around a core user team before wider implementation.

\section{Methods}

Evaluation methodology is used to capture the clinical outcomes and patient/community nurse experience of using Simple Telehealth Florence to manage and monitor a range of health conditions such as diabetes, hypertension, and COPD. This project captures the findings from the first 6 months of activity using a simple, self-completed telehealth satisfaction questionnaire. A convenience sampling approach ${ }^{32}$ was implemented to capture the views of 37 patients and 33 community nurses involved in the project. All patients and staff involved in the study were asked to complete the questionnaire by the Flo team at the end of 6-month contact. The questionnaire was designed locally, and no pilot study was undertaken to test the questions prior to distribution.

The questionnaire involves the patient completing 13 questions on their views of and perceived benefit of "Flo" 
on a five-point Likert scale (ranging from $1=$ strongly agree to $5=$ strongly disagree). Community nurses are then asked to rate the perceived benefit of the system to the patient and their management of the condition on a similar Likert scale. This design has been favored in a number of Telehealth studies, eg, Mair et al ${ }^{33}$ used a simple self-completed questionnaire to evaluate patient and provider responses as part of their randomized controlled trials. Ryan et $\mathrm{al}^{34}$ also adopted a poststudy questionnaire to measure patient satisfaction with their Telehealth intervention.

The approach used in this study enabled a comprehensive response to be collated from both perspectives and supports the idea of co-construction and open transparent relationships between patient and care provider. Permission to access and distribute the questionnaire was obtained from local clinical commissioning groups involved in the roll out of Simple Telehealth: Florence. In this study, the patient was not requested to reflect on changes in nurse interactions or on the quality of nurse treatment received or perceived, but this may be an important and interesting aspect for future studies as SMS texting becomes more widely adopted for patient monitoring.

\section{Results}

The results of this questionnaire are based on 70 completed responses ( $n=37$ patients; and $n=33$ community nurses). Participants in the project represent three localities in the East Midlands region of England, UK. All patients were registered with a general practitioner (GP) and had contact with the general practice team (including the community nurse) on average 1.21 times per month (minimum: zero times; maximum: three times) at the outset of the project. This accounted for health checks, medication adjustments, and exacerbations of the condition. The results show that this decreased to 0.63 times per month following the use of Simple Telehealth Florence for 6 months. Comments from patients indicate an overall agreement that Florence supports their existing care with nurses and doctors and has helped reduce the number of visits they make to their GP. Table 2 summarizes the patients' reported experience of using Simple Telehealth Florence.

Table 2 shows that the majority of patients $(97 \% ; n=36)$ found that it is easy to use a mobile phone to send their vital signs/health information and have no concerns over privacy and sharing information. Some patients dislike the lack of human contact $(14 \% ; n=5)$ and this is known to be a subject of concern for nurses, which arises when Telehealth is proposed. ${ }^{35}$ It may be that this smaller grouping needs assessment for further social contact, social support, and community interventions rather than relying upon nurse visits for socialization. Notably, even in this short study, 94\% of patients felt that the system was already helping them to manage their conditions better. This is an interesting finding even in such a small group and supports the idea that Flo and similar systems may lead to better self-management in the long-term for chronic conditions. The results also show that patients find the system convenient, and that it saves them time. Anecdotal evidence from patient comments is overall in the positive. For example, one patient comments "Flo is really easy to use and is reassuring after a worrying diagnosis"

Table 2 Patient experience of Flo

\begin{tabular}{|c|c|c|c|c|c|}
\hline Patient evaluation $(n=37)$ & Strongly agree & Agree & No change & Disagree & Strongly disagree \\
\hline $\begin{array}{l}\text { Staff can get a good understanding of my health problem } \\
\text { using Florence }\end{array}$ & $20(54 \%)$ & $15(40 \%)$ & $0(0 \%)$ & $2(5 \%)$ & $0(0 \%)$ \\
\hline Florence invades my privacy & I (3\%) & $0(0 \%)$ & $0(0 \%)$ & $17(46 \%)$ & $19(51 \%)$ \\
\hline I feel comfortable using a mobile phone & $19(51 \%)$ & $16(43 \%)$ & $0(0 \%)$ & $0(0 \%)$ & I (3\%) \\
\hline $\begin{array}{l}\text { I feel confident that sending readings to Florence makes } \\
\text { a difference }\end{array}$ & $18(48 \%)$ & $18(48 \%)$ & $0(0 \%)$ & $\mathrm{I}(3 \%)$ & $0(0 \%)$ \\
\hline Florence is helping me manage my own health better & $18(48 \%)$ & 17 (46\%) & I (3\%) & I (3\%) & $0(0 \%)$ \\
\hline Florence is improving my general health & $8(22 \%)$ & $19(51 \%)$ & $3(8 \%)$ & $4(11 \%)$ & $\mathrm{I}(3 \%)$ \\
\hline I dislike the lack of human contact when I use Florence & $\mathrm{I}(3 \%)$ & $4(11 \%)$ & $\mathrm{I}(3 \%)$ & $19(51 \%)$ & II (30\%) \\
\hline I find Florence very convenient & $15(40 \%)$ & $18(48 \%)$ & $\mathrm{I}(3 \%)$ & I (3\%) & I (3\%) \\
\hline Florence saves me time & $12(32 \%)$ & $16(43 \%)$ & $3(8 \%)$ & $4(11 \%)$ & I (3\%) \\
\hline $\begin{array}{l}\text { I feel very reassured that Florence is helping me manage } \\
\text { my health and well-being }\end{array}$ & $16(43 \%)$ & $17(46 \%)$ & $\mathrm{I}(3 \%)$ & $2(5 \%)$ & $\mathrm{I}(3 \%)$ \\
\hline $\begin{array}{l}\text { I believe that Florence should become a standard service } \\
\text { in the future }\end{array}$ & $22(59 \%)$ & $12(32 \%)$ & $0(0 \%)$ & $3(8 \%)$ & $0(0 \%)$ \\
\hline I feel that Florence supports existing care I receive & $21(57 \%)$ & $14(38 \%)$ & $0(0 \%)$ & I (3\%) & $0(0 \%)$ \\
\hline $\begin{array}{l}\text { Regular contact with Florence means I need to visit } \\
\text { my GP less often }\end{array}$ & $17(46 \%)$ & $13(35 \%)$ & $2(5 \%)$ & $4(11 \%)$ & $0(0 \%)$ \\
\hline
\end{tabular}

Abbreviations: GP, general practitioner; Flo, Simple Telehealth "Florence." 
(patient with hypertension). This is supported by Ryan et al, ${ }^{34}$ who noted patients' ease of use of the mobile phone when monitoring asthma. Mair et $\mathrm{al}^{33}$ also add that their patient group consistently demonstrated positive views of telecare in comparison to health care providers.

Several patients ( $n=33 ; 89 \%)$ agree that by sending their health information daily via SMS and following the guidance given by the computerized system, staff get a good understanding of their health problem. However, $8 \%(\mathrm{n}=3)$ disagree that the system helps and $1 \%$ did not notice any change. Those who agree comment that by supplying this information, they can have more meaningful conversations about their health needs when they see a doctor or nurse.

One comment was "I would say that Flo has saved my life, I am no longer going in and out of hospital and when I do see my doctor and nurse I can have a more meaningful discussion about my health and they can see my progress on the computer" [patient with diabetes].

Many patients noticed a reduction in the number of visits to their GP $(81 \% ; n=30)$ and felt reassured that their health was still being monitored. One COPD nurse adds, "Flo reassured the patient that although they were anxious and felt breathless, their vital signs were actually stable and Flo would identify any changes."

All patients would recommend the Simple Telehealth service and echo that it should be a standard service offered to those requiring monitoring of their health at different points in their care plan. From a staff perspective, the majority of clinicians $(84 \% ; n=28)$ involved in the evaluation agreed that Flo helps their patients manage their own health and well-being. Table 3 summarizes the staff experience of using Flo and illustrates that $45 \%(n=15)$ staff report they had fewer contacts with their patient once they started using Flo; $36 \%(n=12)$ saw no change in contact level; and 18\% $(n=6)$ witnessed a slight increase in contact. One specialist nurse reflects on her observations and experiences of working with patients with long-term conditions and states as follows: "There is a culture change that clinicians need to go through. This means we have to stop unnecessary visits to patients and allowing Flo to support patients on our behalf" [COPD nurse].

Another specialist nurse echoes these comments and supports the findings that $54 \%(n=18)$ of nurses felt their patient contact was more appropriate, $24 \%(n=8)$ experienced no change, and 18\% $(n=6)$ had less appropriate contact. Multiple comments from staff perspective highlight that Flo is easy to use and supports their existing approach to care delivery. The following extracts capture this theme from the evaluations.

Flo is working really well - thanks. I have now had to increase one chaps weight readings to three times per week as his weight has gone up since last week, and he is clinically showing signs of deterioration, therefore I need to alter his meds accordingly. Flo means that I don't have to go in three times per week. I still visit once per week, and as he gets worse I will have to go in more, but for now it is really helping me gauge his condition. [Heart failure nurse]

Flo is simple to use and allows my colleagues and I to risk assess our patients prior to surgery. [Pre-op nurse]

\section{Limitations}

A small sample size is acknowledged and expected as part of this interim evaluation. For this stage of local evaluation, it provides a good indicator of patient and staff experience of using the service. The study adopted a locally designed satisfaction survey. No pilot study was undertaken to test the reliability and validity of the tool, thus highlighting this as a key limitation. The design of the survey also aimed to embrace a co-construction approach in terms of patients and staff reflecting on their experience together. Another limitation of this approach is that it could restrict both parties being open and honest about their experience to avoid discomfort or distress to the other. A degree of positivity is highlighted from the evaluation from the patient's perspective and it is acknowledged that those involved in the study opted to

Table 3 Staff evaluation $(n=33)$ of Flo

\begin{tabular}{llllll}
\hline & Definitely yes & Probably yes & No change & Probably no & Definitely no \\
\hline $\begin{array}{l}\text { Do you believe that using Florence has helped } \\
\text { the patients to manage their own health and well-being better }\end{array}$ & $12(36 \%)$ & $16(48 \%)$ & $3(9 \%)$ & $2(6 \%)$ & $0(0 \%)$ \\
& A lot more & A little more & No change & A little fewer & A lot fewer \\
\hline $\begin{array}{l}\text { Have you had more or fewer contacts with this patient since } \\
\text { they started using Florence }\end{array}$ & $0(0 \%)$ & $6(18 \%)$ & $12(36 \%)$ & $13(39 \%)$ & $2(6 \%)$ \\
& Definitely more & Probably more & No change & Probably less & Definitely less \\
\hline $\begin{array}{l}\text { Have your contacts with this patient been more or less } \\
\text { appropriate since they started using Florence }\end{array}$ & $5(15 \%)$ & $13(39 \%)$ & $8(24 \%)$ & $4(12 \%)$ & $2(6 \%)$
\end{tabular}

Abbreviation: Flo, Simple Telehealth "Florence." 
engage with the process and thus may present with increased motivation and ultimately improved satisfaction.

\section{Discussion}

Living with a long-term condition is challenging for patients, particularly to sustain good health and stability against disease progression. Nurses play a key role in the lives of patients through education, support, and monitoring of symptoms and the person's ability to cope. Simple Telehealth: Florence "Flo" not only provides the patient with the opportunity to take responsibility for their health condition, it allows the nurse to collect, monitor, and review the patient's progress over time in a socially unobtrusive yet regular manner. A number of patients talk about being able to have more-meaningful conversations about their life, their illnesses, and the evidence from their health monitoring. Improved quality of life, selfmanagement, and satisfaction with Telehealth is noted in the findings from multiple quantitative and qualitative studies over the past 2 decades ${ }^{28-30,33,34}$

Acceptance and usefulness of the new technology to support patients to self-manage was evident from both patient and staff perspectives. ${ }^{28-30}$ This may be reflective of patients' feeling or being motivated to change their behavior at that time and also influenced by staff interested in using innovative approaches to care delivery. In the literature, a number of authors remark that successful adoption of new approaches to care relies on innovators and early adopters to drive forward the cultural change. ${ }^{36}$ The use of a Telehealth system such as Flo does not eradicate the role of health professionals in the management of long-term conditions but supports existing care delivery approaches, allowing nurses to safely manage at a distance and helps to avoid unnecessary visits. The project has also shown that this can provide reassurance to patients that their symptoms are being regularly assessed and monitored. One nurse commented that community nurses need to develop confidence in their patients to self-manage and this involves a culture change. This is also mirrored in the literature as a means of letting go of the power between patient and nurse and the wider cultural/organizational issues around acceptance and usefulness of innovative technologies. ${ }^{10,36}$

Overall, the patient and staff experience of using Flo is positive and mutual benefits are evidenced in terms of time, convenience, and ease of use. The results are small in magnitude but provide insight into the lives of patients, their satisfaction with using Telehealth, and the use and acceptance of the model to complement existing self-management strategies by nurses in primary care services.

\section{Conclusion}

This evaluation captured the experiences of patients and staff using a simple, yet innovative way for patients and community nurses to work collaboratively while supporting the principles of unobtrusive distance monitoring that may encourage better self-management. The findings around the use of Flo are positive and support the continued rollout of this system with a view to sustaining and embedding Flo within this region of NHS England. Flo is available across NHS England and can be used by nurses in a number of ways, eg, in smoking cessation, weight management clinics, or, as seen in this study, to monitor long-term conditions. As shown here, small-scale evaluations can substantiate a number of benefits and help understand the patient's experiences of living with and monitoring their long-term condition. Importantly, these small-scale evaluations allow a better-managed change process by which the local clinical staff can use, interact with, and comment on a new system in collaboration with their patients and this may help to reduce barriers to change.

\section{Implications for practice}

Flo offers nurses a simple yet innovative way to assess, monitor, and evaluate a patient's health, lifestyle, and behavior. This has the potential to change the way in which consultations are structured and facilitated for some patients (but not all) and whereby staff can view the information provided by their patient over time, eg, blood glucose results, thus releasing time for the nurses to consider other therapeutic interventions and approaches to support the individual.

Embedding Flo as an option within primary care services within the community is proposed as a recommendation to practice. In this context, it is important that Flo is considered alongside other diagnostic, therapeutic, and administrative care management systems as a simple, flexible, and rapidly deployable tool when someone requires short- or long-term monitoring of a health condition.

The authors hope that this article will encourage clinical teams to reflect on their approach to home and community monitoring of patients, encouraging better self-management, and that clinical staff will consider how and where Flo could support patient groups in line with national protocols.

\section{Acknowledgment}

The authors thank the East Midlands Academic Health Science Network (EMAHSN), which funded the interim evaluation of Simple Telehealth "Flo" (http://www.emahsn. org.uk). 


\section{Disclosure}

The authors report no conflicts of interest in this work.

\section{References}

1. Christensen K, Doblhammer G, Rau R, Vaugel J. Ageing population: the challenges ahead. Lancet. 2009;374(9696):1196-1208.

2. Reeves D, Kennedy A, Fullwood C, et al. Predicting who will benefit from an expert patient s programme self-management course. Br J Gen Pract. 2008;58(548):198-203.

3. Randall S, Daly G, Thunhurst C, Mills N, Guest DA, Barker A. Case management of individuals with long term conditions by community matrons: report of qualitative findings of a mixed method evaluation Primary Health Care Res Dev. 2014;15:26-37.

4. Department of Health Digital Health Strategy. 2013. Available from: https://digitalhealth.dh.gov.uk/digital-strategy/executive-summary/. Accessed March 18, 2014.

5. Scottish Government. A National Telehealth and Telecare Delivery Plan for Scotland to 2015: Driving Improvement, Integration and Innovation [online]; 2013. Available from: http://www.scotland.gov. uk/Publications/2012/12/7791/2. Accessed December 4, 2013.

6. Welsh Government. More than Just Words: A Strategic Framework for Welsh Language Services in Health, Social Services and Social Care. Cardiff: Welsh Government; 2012.

7. Thompson DR, Clark AM. The complexity conundrum nursing interventions for complex health problems. Contemp Nurse. 2012;40(2): 277-280.

8. Griffiths C, Foster G, Ramsay J, Eldridge S, Taylor S. How effective are expert patient education programmes for chronic disease? $\mathrm{Br}$ Med J. 2007;334:1254-1258.

9. Lorig K, Holman H. Self-management education: history, definition, outcomes and mechanisms. Annual Behav Med. 2003;26(1):1-7.

10. McWilliam CL. Patients, persons or partners? Involving those with chronic disease in their care. Chronic Illn. 2009;5:277-287.

11. Kirby SE, Dennis SM, Bazeley P, Harris MF. What distinguishes clinicians who better support patients for chronic disease management? Australian J Primary Health. 2012;18(3):220-227.

12. Wilson PM, Kendall S, Brooks F. Nurses' responses to expert patients, the rhetoric and reality of self-management in long term conditions: a grounded theory study. Int J Nurs Stud. 2006;43:803-818.

13. Paterson GA, Nayda RJ, Paterson JA. Chronic condition self-management: working in partnership toward applied models for age and culturally diverse clients. Contemp Nurse. 2012;40(2):169-178.

14. Shen H, Edwards H, Courtney M, McDowell J, Wei J. Barriers and facilitators to diabetes self-management: perspectives of older community dwellers and health professionals in china. Int J Nurs Pract. 2012;19(6):627-635.

15. Rodgers A, Gately C, Kennedy A, Sanders C. Are some more equal than others? Social comparison in self-management skills training for long term conditions. Chronic Illn. 2009;5:305-317.

16. TSA. What is Telehealth? [online]; 2013. Available from: http:// www.telecare.org.uk/consumer-services/what-is-telehealth. Accessed August 28, 2014

17. Wootton R. Twenty years of telemedicine in chronic disease management - an evidence synthesis. J Telemed Telecare. 2012; $18: 211$.

Nursing: Research and Reviews

\section{Publish your work in this journal}

Nursing: Research and Reviews is an international, peer-reviewed, open access journal publishing original research, reports, reviews and commentaries on all aspects of nursing and patient care. These include patient education and counselling, ethics, management and organizational issues, diagnostics and prescribing, economics and
18. Greenhalgh T, Procter R, Wherton J, Sugarhood P, Shaw S. The organising vision for telehealth and telecare: discourse analysis. $B M J$ Open. 2012;2:e001574.

19. Jennett PA, Affleck Hall L, Hailey D, et al. The socio-economic impact of Telehealth: a systematic review. J Telemed Telecare. 2003;9:311.

20. Sood S, Mbarika V, Jugoo S, et al. What is telemedicine? A collection of 104 peer reviewed perspectives and theoretical underpinnings. Telemed J E Health. 2007;13:5.

21. Wallwiener M, Wallwiener CW, Kansy JK, Seeger H, Rajab TK. Impact of electronic messaging on the patient-physician interaction. J Telemed Telecare. 2009;15:243.

22. McLean S, Nurmatov N, Liu JLY, Pagliari C, Car J, Sheikh A. Telehealthcare for chronic obstructive pulmonary disease: cochrane review and meta-analysis. Br J Gen Practice. 2012;62:e739.

23. Doughty K. SPAs (smart phone applications) - a new form of assistive technology. J Assistive Technol. 2011;5(2):88-94.

24. Young C, and Wilkins, A. 2011, Digital first: The delivery choice for England's population. Transform Innovation Ltd.

25. Neilson. Neilson: The Mobile Consumer: A Global Snap Shot [online]. 2013. Available from: http://www.nielsen.com/content/dam/./MobileConsumer-Report-2013.pdf. Accessed September 11, 2014.

26. Scottish Government 2011. Scotlands Digital Future [online]; 2014. Available from: http://www.scotland.gov.uk/Publications/. Accessed July 22, 2014.

27. Department of Health. Digital Strategy: Leading the Culture Change in Health and Care [online]; 2012. Available from: http://www.dh.gov. uk/publications. Accessed July 22, 2014.

28. DeJongh T, Gurol-Urganci I, Vodopivec-jamesek V, Car J, Atun R. Mobile Phone Messaging for Facilitating Self-Management of Long Term Illness (Review). Oxford: Cochrane Collaboration, John, Wiley and Sons Ltd; 2012.

29. Cottrell E, Chambers R, O'Connell P. Using simple Telehealth in primary care to reduce blood pressure: a service evaluation. BMJ Open. 2012;2:e001391.

30. Cottrell E, McMillan K, Chambers R. A cross sectional survey and service evaluation of simple Telehealth in primary care: what do patients think? BMJ Open. 2012;2:e01392.

31. Chambers R. Tackling Telehealth: How CCG's Can Commission Successful Telehealth Services: Inside Commissioning in association with Simple Telehealth; 2014. Available from: https://www. insidecommissioning.co.uk. Accessed March 27, 2014.

32. Liobiondo-wood G, Laber J. Nursing Research: Methods for Critical Appraisal for Evidence Based Practice. 8th ed. Mosby: Elsevier; 2013.

33. Mair FS, Goldstein P, May C, et al. Patient and provider perspectives on home telecare: preliminary results from a randomised controlled trial. J Telemed Telecare. 2005;11:95-97.

34. Ryan D, Cobern W, Wheeler J, Price D, Tarassenko L. Mobile phone technology in the management of asthma. JTelemed Telecare. 2005;11: 43-46.

35. Brewster L, Mountain G, Wessels B, Kelly C, Hawley M. Factors affecting frontline staff acceptance of Telehealth technologies: a mixed method systematic review. J Adv Nurs. 2013;70(1):21-23.

36. Llewellyn S, Procter R, Harvey G, Maniatopoulos G, Boyd A. Facilitating technology adoption in the NHS: negotiating the organisational and policy context: a qualitative study. Health Serv Delivery Res. 2014;2:23.

37. Chambers R. Simple telehealth SMS texting service: Flo clinical protocols AIM for Health version 2. NHS Stoke-on-trent Clinical Commissioning Group. 2013.

\section{Dovepress}

resource management, health outcomes, and improving patient safety in all settings. The manuscript management system is completely online and includes a very quick and fair peer-review system. Visit http://www.dovepress.com/testimonials.php to read real quotes from published authors. 